\title{
LES WITH ACOUSTICS AND FSI FOR DEFORMING PLATES IN GAS FLOW
}

\author{
I1. Per Nilsson, I2. Eric Lillberg and I3. Niklas Wikström \\ $\AA$ A $-T \ddot{U} V$ Nord, Sweden \\ I2. presently at Westinghouse \\ I3. presently at FS Dynamics
}

\begin{abstract}
This concerns Flow Induced Vibrations (FIV) in nuclear reactors and numerical analysis of such. Special attention is paid to structural excitation by sound generated remotely and turbulent flow around the structure. One hypothesis was that these phenomena can interact, so that the structure accumulates more energy from the flow if it also excited by sound from another source. In the studies, Fluid-Structure Interaction (FSI) is simulated with Large Eddy Simulations (LES).
\end{abstract}

It is shown possible to simulate excitation due to both acoustic and turbulence loads using the reported methods, at least qualitatively. The excitation levels are even of the right order of magnitude in some parts. However, there are some shortcomings in the modeling. The most important is perhaps the lack of non-reflecting boundary conditions. Another problem is the strong numerical damping in combination with demanding numerics for the selected solid solution methodology.

Three cases are simulated, two for validation and one applied about steam dryers. For the applied case, it is concluded unlikely that excitation by the acoustic and turbulence loads can interact. The main reason is that the flow is controlled more by static geometrical factors, such as solid rotation sharp edges, than small deformations due to vibrations.

\section{INTRODUCTION}

The work is carried out by CFD in open source software including FSI. It is necessary to test and validate the calculation methods for this study. The study also includes testing of advanced methods for flow simulation, such as LES.

To validate the methods, two cases have been investigated. In Case 1 it is studied how a structure can be excited by an acoustic load without flow. In Case 2 it is studied how a structure can be excited by flow without acoustic loading. In Case 3 a practical problem with steam dryer structures is studied.

\section{METHODS}

A new general solver for transient FSI problems such as FIV has been implemented by carefully combining algorithms for transient flow and structural analysis. The algorithms used are available in the official distribution of OpenFOAM-1.5.x (2009). The new solver is called mRCFF here.

For the flow, a compressible pressure based LES solver is used without explicit filtering, that is, the mesh is used as a filter. This is a simple solution that could be considered industry standard, despite (or perhaps because) its shortcomings are well known. Here the Smagorinsky SGS-model is used and the convective terms are discretized on a second order centered stencil with some limiting.

For the structure, a linear elastic transient solution algorithm is used. Both are based on finite volumes, which is a somewhat unconventional choice for the structural dynamic simulation. Separate systems of equations are created in the regions for fluid and solid. Each region has its own mesh, numerics and custom fields, in which the boundary conditions, which allow communication between the different regions, are located. Parallelization is done by domain separation of the various regions.

In the cases analyzed here explicitly coupled FSI algorithms are used on conformal meshes. These generally utilize a much finer network than justified by the FSI problem and thus give longer solution 
times for the structural problem. This method however allows for easier parallelization. Both solvers work on deforming meshes. The deformation of the fluid mesh is given implicitly by the solution of the displacement field in the structure solver. A separate displacement field solver is used to propagate the deformation of fluid mesh nodes. The movement is done with a diffusion which is proportional to the square of the distance to the fluid/structure-edge. This gives a fairly local deformation of the fluid network that preserves the network structure.

\section{CASE 1}

In Case 1 it is tested how the structural excitation by an external sound source can be simulated with mRCFF. Measurements and calculations by Frendi and Robinson (1993) have been selected for comparison. The case is formulated in such a way that comparisons can be two-dimensional.

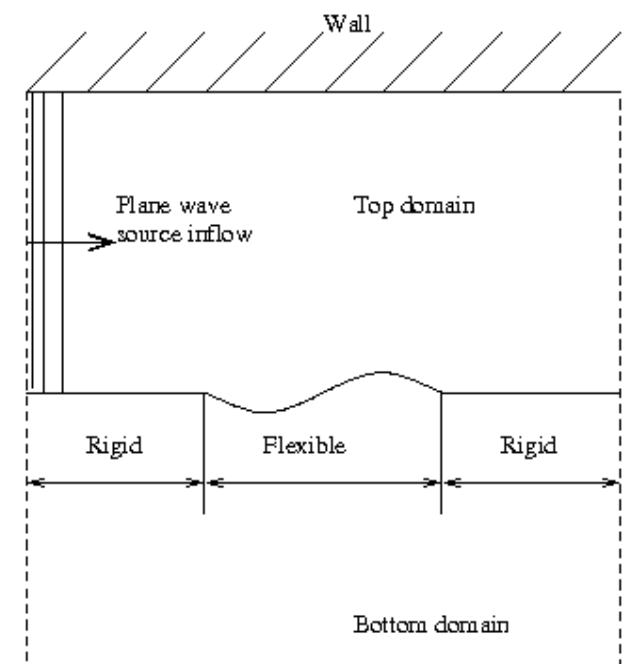

Figure 1: Domains according to Frendi and Robinson (1993)

The upper domain dimensions are $\mathrm{B}=0.2794 \mathrm{~m}, \mathrm{H}_{\text {top }}=0.3048 \mathrm{~m}, \mathrm{~L}=3.048 \mathrm{~m}$. The plate dimensions are $\mathrm{L}_{\mathrm{p}}=0.381 \mathrm{~m}, \mathrm{t}_{\mathrm{p}}=3.302 \mathrm{~mm}$. The air properties are $\mathrm{T}_{\mathrm{a}}=288.33 \mathrm{~K}, \rho_{\mathrm{a}}=1.2258 \mathrm{~kg} / \mathrm{m}^{3}, \mathrm{p}_{\mathrm{a}}=101325 \mathrm{~Pa}, \mathrm{c}_{\mathrm{a}}$ $=340.16 \mathrm{~m} / \mathrm{s}, \mathrm{C}_{\mathrm{va}}=717.4 \mathrm{~J} /(\mathrm{kgK}), \kappa_{\mathrm{a}}=1.4$ and the plate properties are $\mathrm{E}_{\mathrm{p}}=3.7546^{*} 10^{10} \mathrm{~Pa}, \mathrm{v}_{\mathrm{p}}=0.3, \rho_{\mathrm{p}}$ $=1816.8 \mathrm{~kg} / \mathrm{m}^{3}, \Gamma_{\mathrm{p}}=6.7862 \mathrm{Ns} / \mathrm{m}^{3}$ (The damping is not modeled here though).

\subsection{Numerical model}

The bottom air domain shown in Figure 1, the cavity, has been omitted in these simulations. Instead constant atmospheric pressure is applied at the lower side of the flexible plate. The mesh consists of 4365 volumes for the air and 95 volumes for the plate. The air volumes are $20 \mathrm{~mm}$ long and high. With a time step of $1 \mathrm{e}-5 \mathrm{~s}$ and the speed of sound $340 \mathrm{~m} / \mathrm{s}$ this corresponds to a CFL-number of 0.17 . The plate volumes are of the same length as the air volumes and $0.66 \mathrm{~mm}$ high. The depth is the same width as the domain, $0.2794 \mathrm{~m}$, because the case is handled as two-dimensional.

The sides of the domain have symmetry boundary conditions for both air and plate. The ceiling and floor outside the plate are walls. The upper surface of the plate is a moving wall with non-slip to the air and pressure and shear stress conditions to the solid. The front and rear end of the plate have displacement 0 in all directions. At the inlet, pressure and velocity are applied as a function of time. The outlet is a convection boundary condition, which lets parallel waves out without reflection. To avoid disturbance from the outlet boundary, the outlet has an extra long outlet section added. This works in practice as a non-reflecting boundary condition as long as the calculation time is shorter than the arrival time of the reflections.

For the spectrum tests, the inlet pressure and velocity are applied as a function of time. The pressure function was generated in the same way as in Frendi and Robinson (1993). A random pressure signal 
with specified sound pressure level (SPL) was first generated. Then it was filtered with a second-order band-pass filter between 50 and $500 \mathrm{~Hz}$. The corresponding velocity function is estimated as pressure change divided by impedance, which is the density times the speed of sound.

\subsection{Test with step load}

As first tests, before the spectrum tests, a step load in pressure was applied at the inlet. These tests are designed to compare the dynamic response of the structure due to a single well-defined load. Initially there is atmospheric pressure and the plate is unloaded. Then at the time of $0.01 \mathrm{~s}$, the pressure is increased $10 \mathrm{~Pa}$ in $0.1 \mathrm{~ms}$. At the same time, the velocity is increased as calculated with the impedance, in the same way as for the random signal described above.
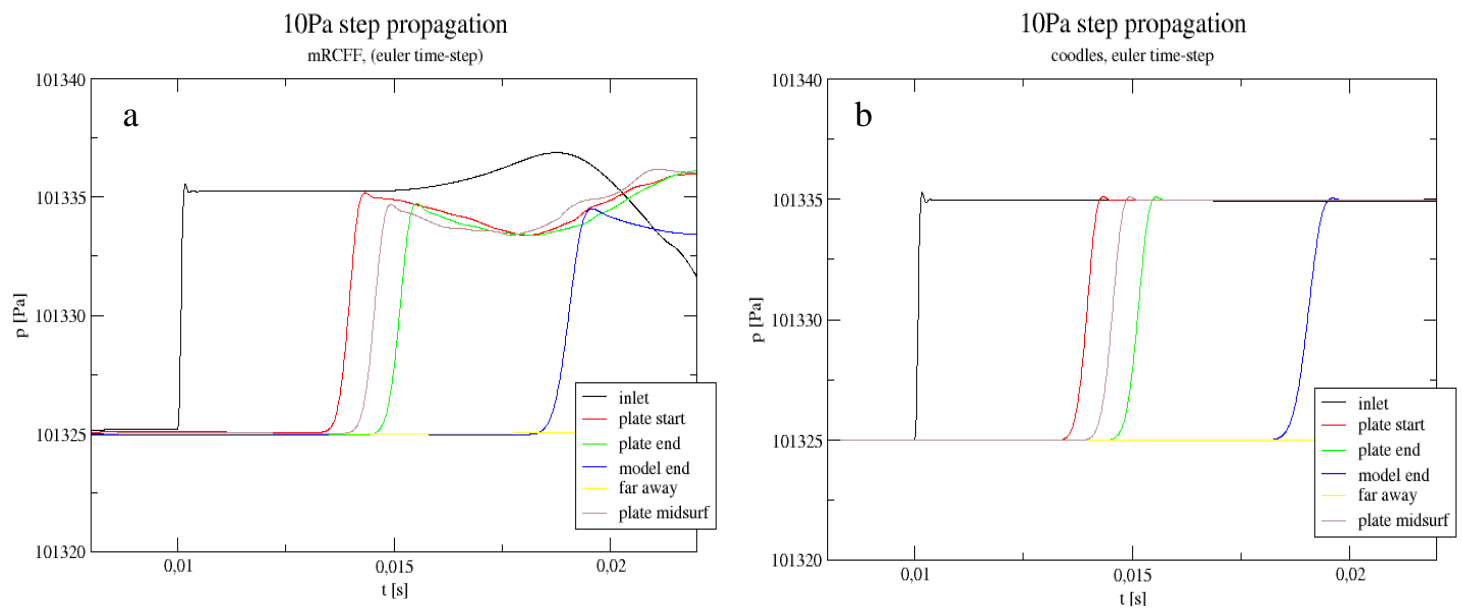

Figure 2: Pressure at different positions with different methods

In Figure 2a a flexible plate with FSI is included in the model. The pressure is affected significantly by the plate deflection. The plate has been modeled as rigid (no FSI) in Figure 2b. Some other available schemes were also tested with this configuration, but all those results are not shown here. With a firstorder Euler-scheme, there was some numerical diffusion. In an analytic solution, the step propagates without deformation, but here the diffusion can be seen in Figure $2 \mathrm{~b}$. With backward-scheme there was dispersion of the step edge, while the Crank-Nicholson 0.9 gave dispersion in both the front and rear. It was concluded that we had to accept the diffusion of the Euler-scheme for this application. The dispersion of the backward-scheme was unacceptable due to the reflecting boundary conditions. The Crank-Nicholson scheme did not work with moving meshes in the present software release.
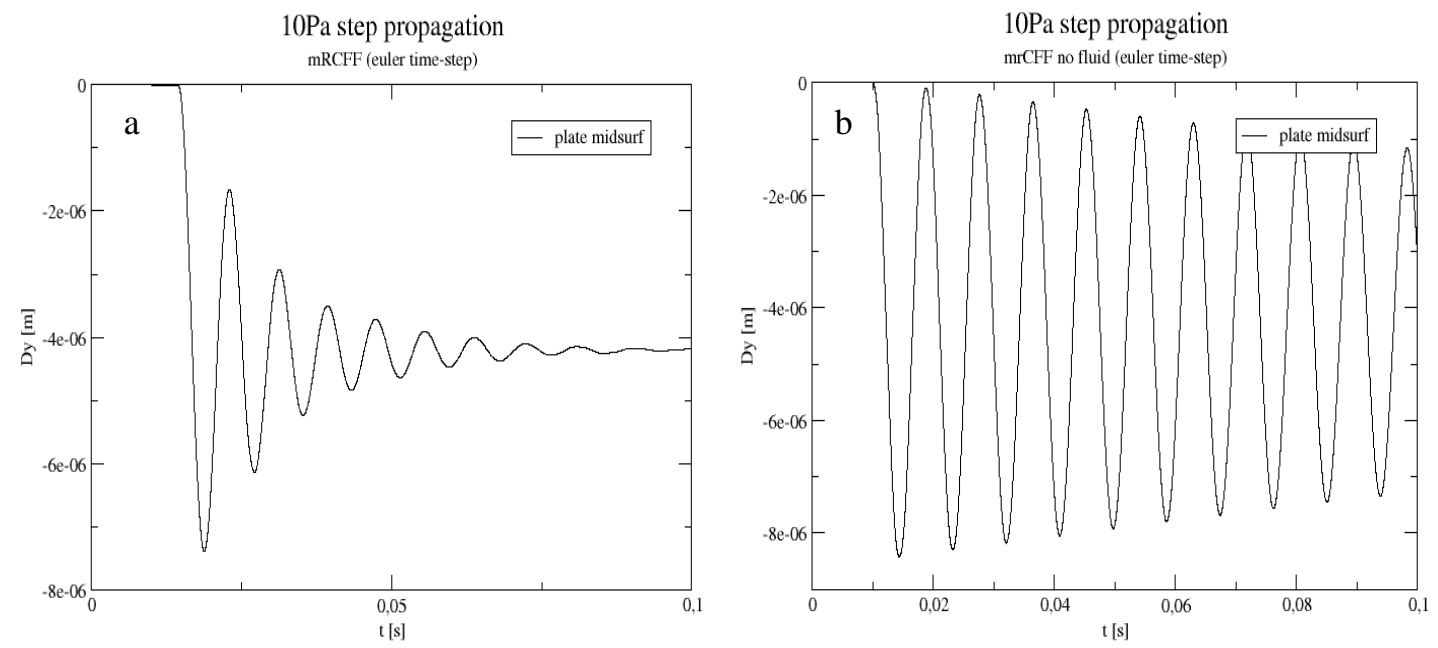

Figure 3: Displacement at plate centre with different methods 
Figure 3 shows plate centre displacement during loading steps. Figure 3a shows the deflection when the air is included in the model. Figure $3 \mathrm{~b}$ shows the deflection when the pressure is placed directly on solid and no air is included in the model. The air damping is clear in Figure $3 a$ and the numerical damping can be seen in Figure 3b. There is no modeled damping in the solid.

It appears also that the deflection converges towards a stationary value of about $4.2 \mathrm{e}-6 \mathrm{~m}$, which is consistent with the handbook formulas for $10 \mathrm{~Pa}$ static load. The natural frequency of the plate is about $112 \mathrm{~Hz}$ from the handbook formulas and linear approximation, which is also simulated quite well.

\subsection{Spectrum tests}

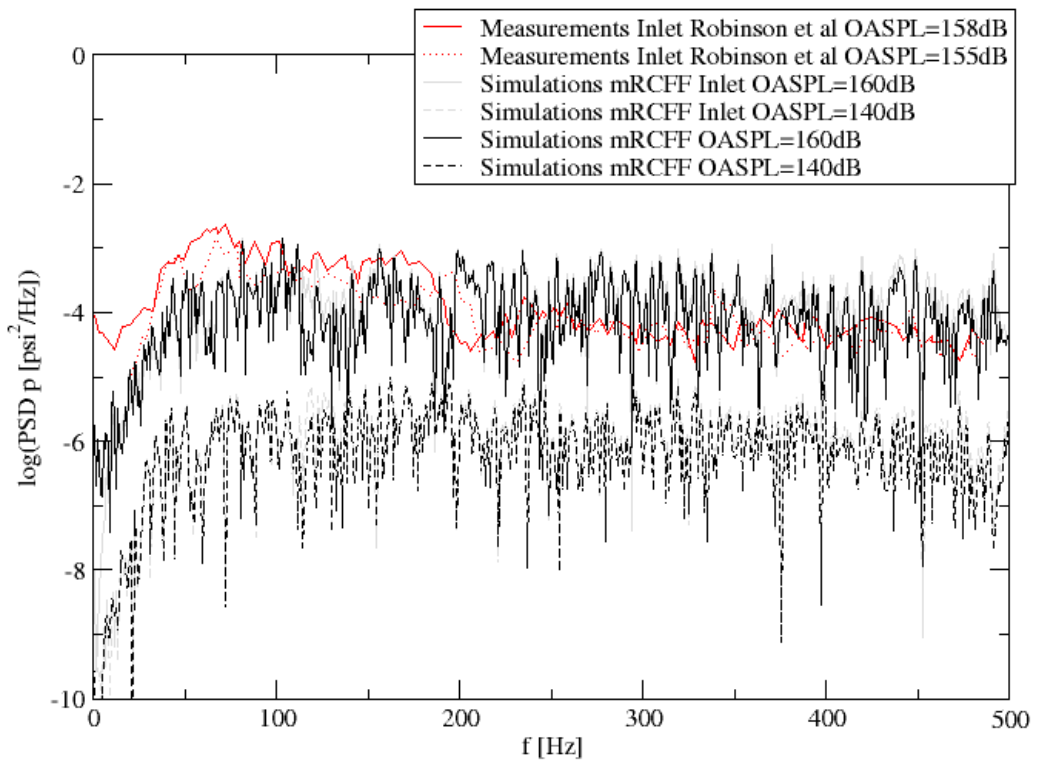

Figure 4: PSD of pressure

The pressure on the plate is strongly governed by pressure at the inlet. In Figure 4 it can be seen that the pressure on the plate agrees well with the pressure measured by Robinson et al (1992) at the inlet. This can also be seen when comparing references Frendi and Robinson (1993) and Robinson et al (1992). The pressure at the inlet of the simulations (which is set as boundary condition) can be seen as gray in the background in the figure.

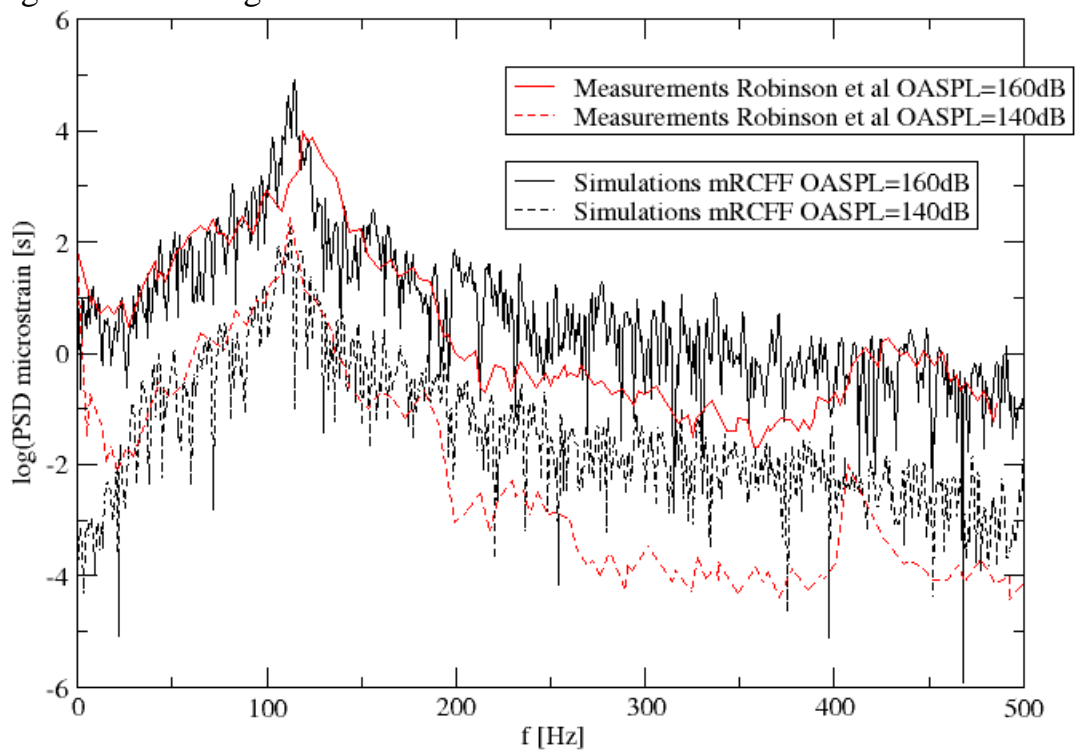

Figure 5: PSD of microstrain, that is strain times 1e6 
In Figure 5 it can be seen that the simulated strain spectra agree well with the strain measured by Robinsonet al (1992), especially around the first eigen-frequency, $112 \mathrm{~Hz}$. At $160 \mathrm{~dB}$, simulations overestimate the frequency of the maximum excitation slightly, while the height is somewhat underestimated. The simulations in Frendi and Robinson (1993) show the same differences. This may be due to differences in damping in the plate and the clamping. According to Frendi and Robinson (1993), the accuracy of the gauges is less good at the low strain levels occurring outside the region around the first eigenfrequency.

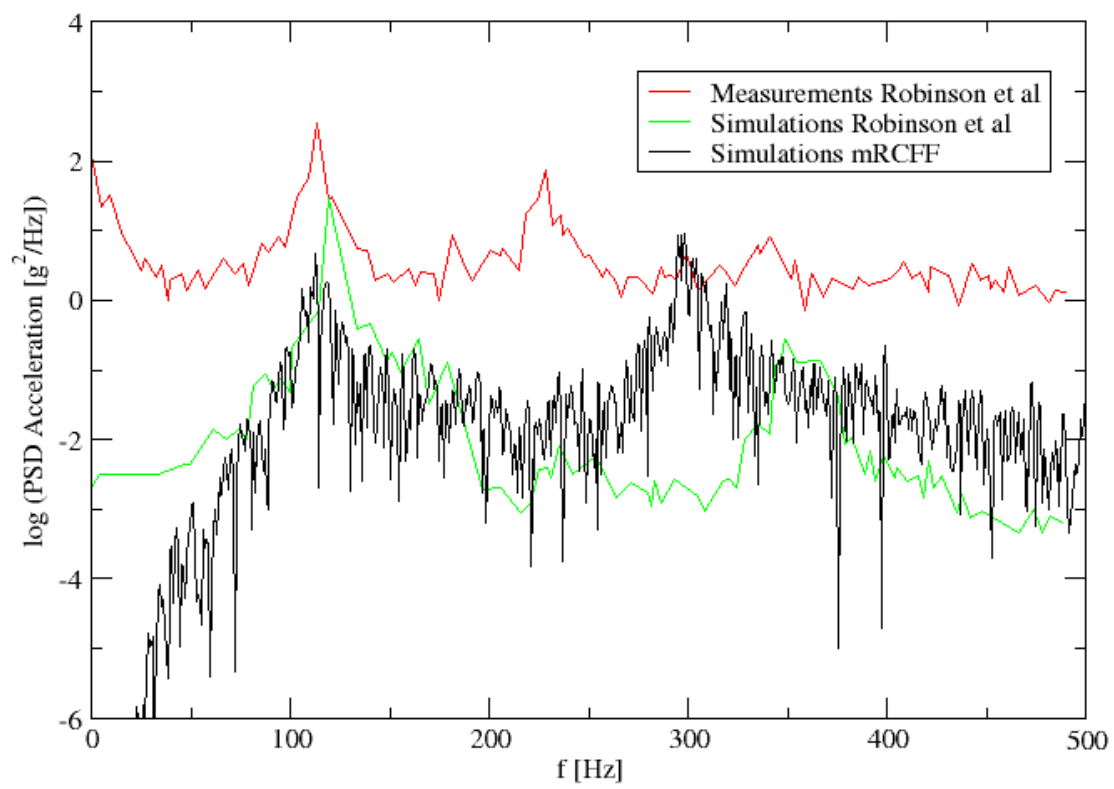

Figure 6: PSD of acceleration

In Figure 6 it can be seen that the simulated accelerations are well below the measured. This is mainly due to problems in the measurements according to Robinson et al (1992). The simulations with mRCFF, however, compare rather well with the simulations by Frendi and Robonson (1993), at least in basics. The second eigenmode is simulated at different frequencies here with mRCFF (around $300 \mathrm{~Hz}$ ) and by Robinson et al (about $350 \mathrm{~Hz}$ ). It might be due to differences in clamping, which is fixed with mRCFF, but has finite stiffness in Robinson et al. There is also some uncertainty about the probe placement. (The differences in clamping stiffness cannot be seen above in Figure 4 and Figure 5 , because they are measured at points where the second mode gives small amplitude.)

\subsection{Assessment case 1}

The methods are assessed as sufficient to study how a structure is affected by acoustic loads phenomenologically and to an order of magnitude. The excitation of the plate in the case of an acoustic wave agrees well with measurements and simulations in Frendi and Robinson (1993) and Robinson (1992). The differences are assessed to be mainly due to differences in damping.

\section{CASE 2}

In Case 2, structural excitation by turbulent flow near a structure is simulated with mRCFF. The case is similar to Case 1 in that there is an inlet on the left end of a rectangular air domain with a plate over a cavity in the bottom. Here it is a fluid inlet instead of an inlet for acoustic waves. There is also a thin bar in front of the plate, see Figure 7. This bar disturbs the flow and creates a turbulent shear layer over the plate. This case is taken from Verge et al (2007) which use measurements form Leclercq (1999). 


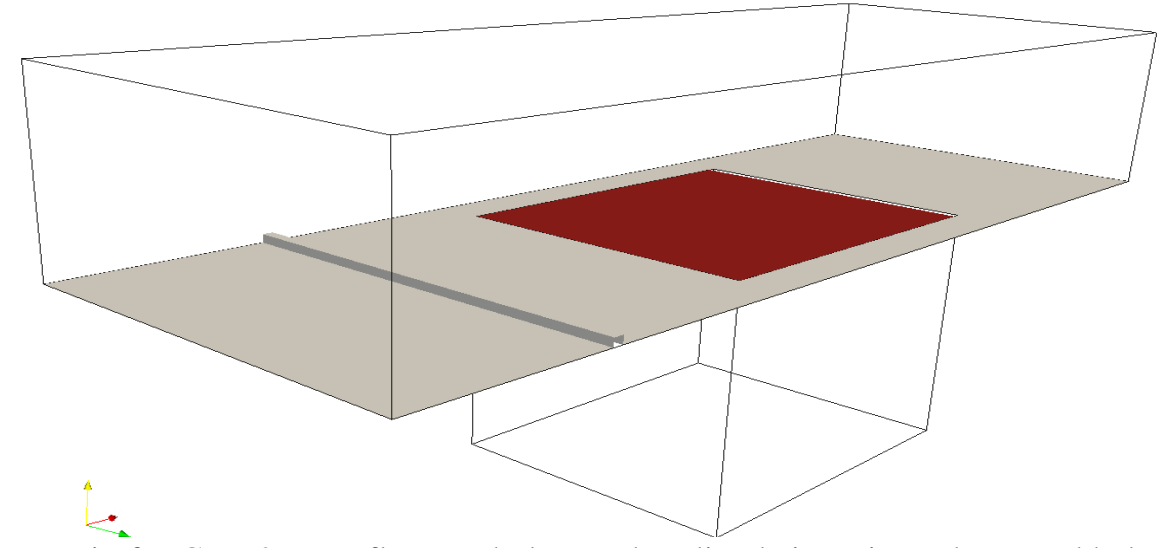

Figure 7: Domain for Case 2, gray floor, red plate and outlined air regions above and below the plate

The upper domain dimensions are $\mathrm{B}=0.396 \mathrm{~m}, \mathrm{H}_{\text {top }}=0.2 \mathrm{~m}, \mathrm{~L}=0.7 \mathrm{~m}$. The lower domain, the cavity, dimensions are $\mathrm{B}=0.306 \mathrm{~m}, \mathrm{H}_{\text {cav }}=0.28 \mathrm{~m}, \mathrm{~L}=0.366 \mathrm{~m}$. The plate dimensions are $\mathrm{L}_{\mathrm{p}}=0.366 \mathrm{~m}, \mathrm{t}_{\mathrm{p}}=1 \mathrm{~mm}$, $\mathrm{b}=0.306 \mathrm{~m}$ and the bar dimensions and location are before plate are $1=10 \mathrm{~mm}, \mathrm{~h}=9 \mathrm{~mm}, \mathrm{a}=-0.2 \mathrm{~m}$.

The air properties are $\mathrm{T}_{\mathrm{a}}=293 \mathrm{~K}, \rho_{\mathrm{a}}=1.19 \mathrm{~kg} / \mathrm{m}^{3}, \mathrm{p}_{\mathrm{a}}=0.1 \mathrm{MPa}, \mathrm{c}_{\mathrm{a}}=344 \mathrm{~m} / \mathrm{s}, \mathrm{C}_{\mathrm{va}}=719 \mathrm{~J} /(\mathrm{kgK}), \kappa_{\mathrm{a}}=1.4$ and the plate properties are $\mathrm{E}_{\mathrm{p}}=210^{*} 10^{11} \mathrm{~Pa}, \nu_{\mathrm{p}}=0.3, \rho_{\mathrm{p}}=7800 \mathrm{~kg} / \mathrm{m}^{3}$.

\subsection{Numerical model}

The air mesh over the plate consists of $1.2 \mathrm{M}$ volumes, the plate of $0.04 \mathrm{M}$ and the air in the cavity under the plate of $0.2 \mathrm{M}$ volumes. In the first layer over the plate, the volumes are $3.7 \mathrm{~mm}$ long and wide and $0.18 \mathrm{~mm}$ high. With time step $1 \mathrm{e}-5 \mathrm{~s}$ and the speed of sound $344 \mathrm{~m} / \mathrm{s}$ this corresponds to an acoustic CFL-number of 0.9 in the longitudinal direction. The convective CFL's are several times smaller. In the plate, the volumes have the same length and width as in the air and are $0.2 \mathrm{~mm}$ high.

For solver reasons, the air above the plate is connected with the cavity via a two volumes long slit at the plate trailing edge. It might be seen in Figure 7 with some effort. The sides of the domain, that is in depth, and ceiling and floor around the plate, are walls with slip condition. The upper surface of the plate is a moving wall with non-slip to the air and pressure and shear stress conditions to the solid. At the plate edges, displacement is set to 0 in all directions. At the inlet, a velocity of $40 \mathrm{~m} / \mathrm{s}$ is applied. The outlet is a convective boundary condition which lets parallel waves pass out without reflection.

\subsection{Turbulent structures and displacement}

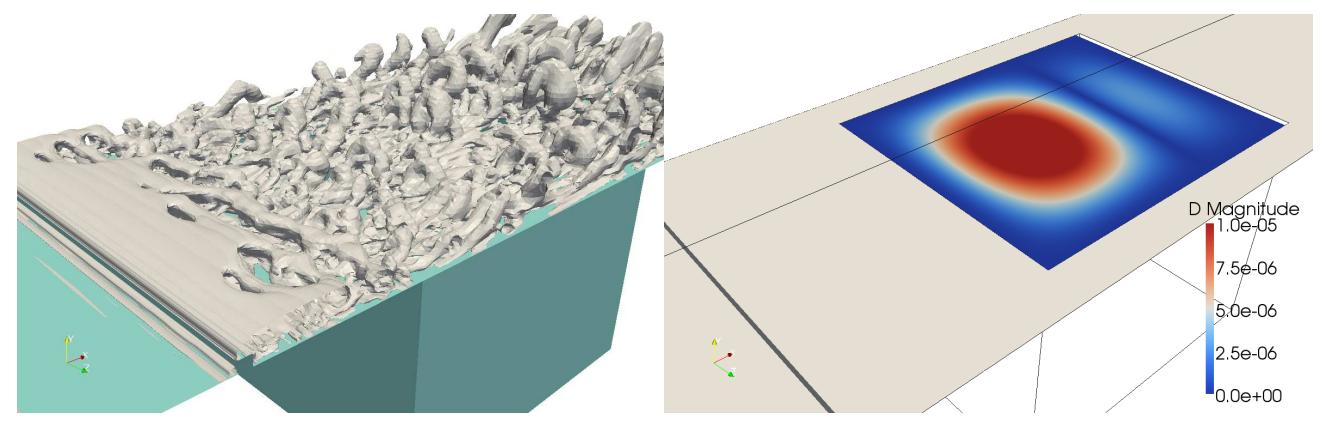

Figure 8: Isosurfaces of $\mathrm{Q}=1000$ (left) and plate displacement (right)

In Figure 8 (left) it can be seen how a horizontal shear layer is formed after the bar. The layer breaks up in rolls and then the rolls are broken up into smaller pieces, which are subsequently deformed to hair-pin shaped structures. The centers of these structures lift up against the free stream, while the ends are stretched along with the flow and thinned because of the stretch.

To the right in Figure 8, it can be seen how the plate deforms in what looks like the first and second mode shapes. The slit at the plate's trailing edge is also visible. 


\subsection{Pressure and acceleration}

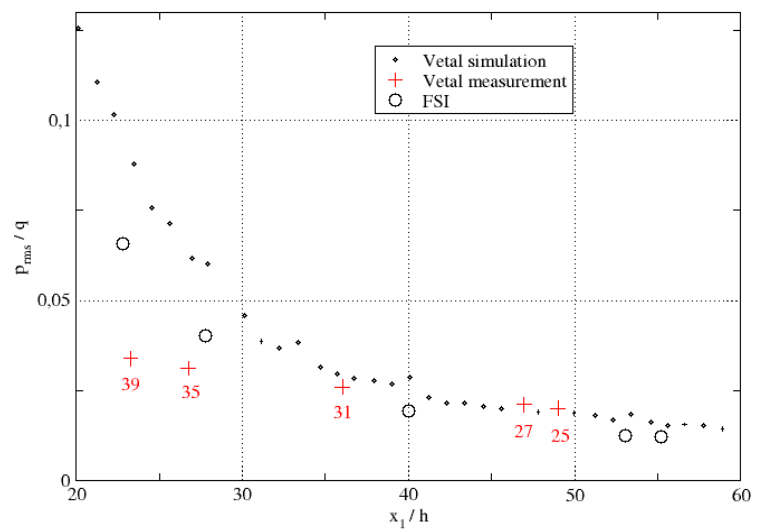

Figure 9 Pressure fluctuations scaled with dynamic pressure along the plate

(Vetal $=$ Vergne et al (2007), FSI $=$ Simulations with $\mathrm{mRCFF}$ )

Figure 9 shows that the simulated pressure fluctuations along the plate compare well to simulations and measurements from Vergne et al (2007) and Leclercq (1999).

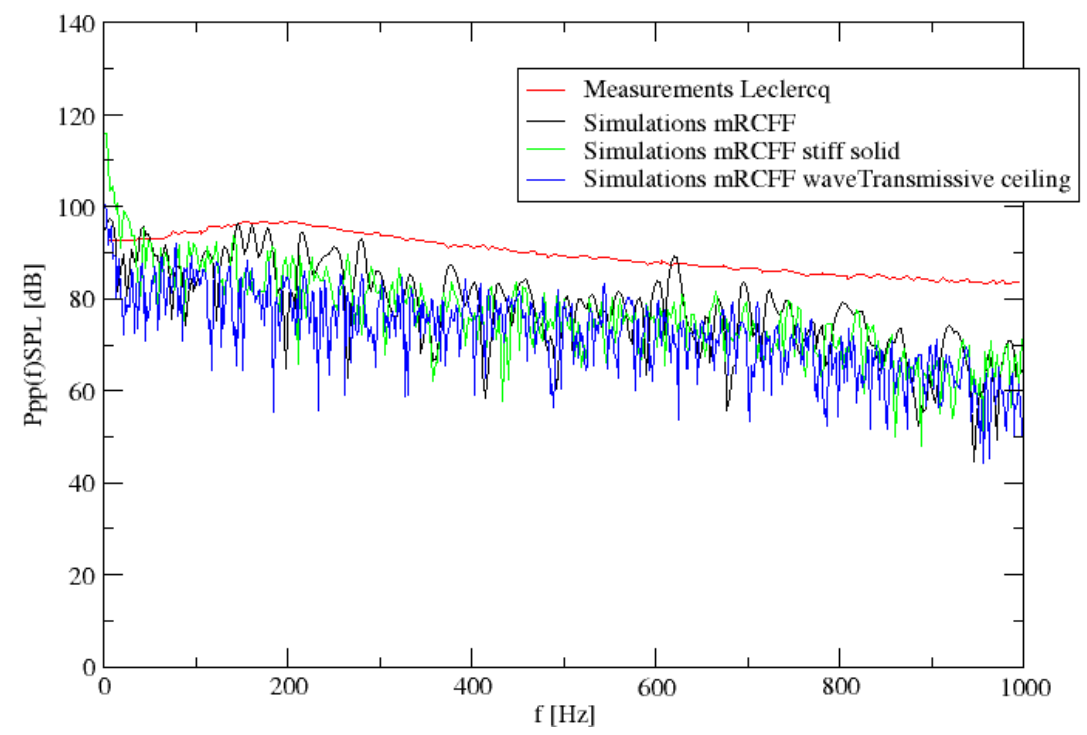

Figure 9: Power spectrum of pressure before plate centre, SPL with reference 2e-5Pa

( Vetal = Vergne et al (2007))

The pressures from the mRCFF simulations are slightly below the measurements by Robinson et al (1992), see Figure 9. The shape of the spectra is similar though, with a peak slightly below $200 \mathrm{~Hz}$.

The black curve is simulated with elastic plate and the green curve is simulated with rigid plate. The small difference between black and green at low frequencies thus represents the impact of the structural motion. The fact that the curves for both rigid and flexible flat plate are at the same level, compared with the measurements, can be interpreted that deviations in the solid model, for example numerical damping, are secondary in this comparison of pressure.

The blue curve is simulated with convection boundary conditions at the ceiling of the domain. In the other simulations, a wall boundary condition is used at the ceiling of the domain. The difference is small, which indicates that the boundary condition at the ceiling does not have much effect. 


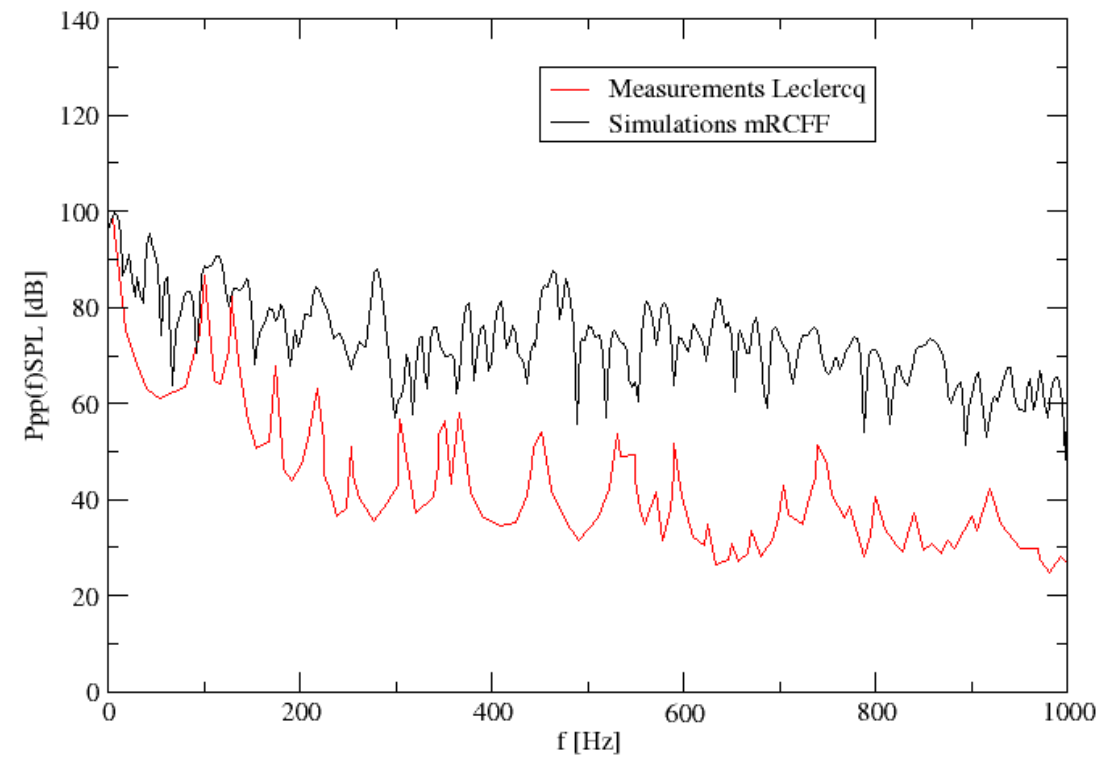

Figure 10: Power spectrum of pressure at the cavity bottom

In Figure 10 it can be seen that the pressure levels in the cavity are well above the levels measured by Leclercq (1999). It is probably due to the slot after the plate, which is modeled in the simulation mRCFF, but that does not exist in the experiments. The slot allows sound energy to get past the plate into the cavity in the simulation.

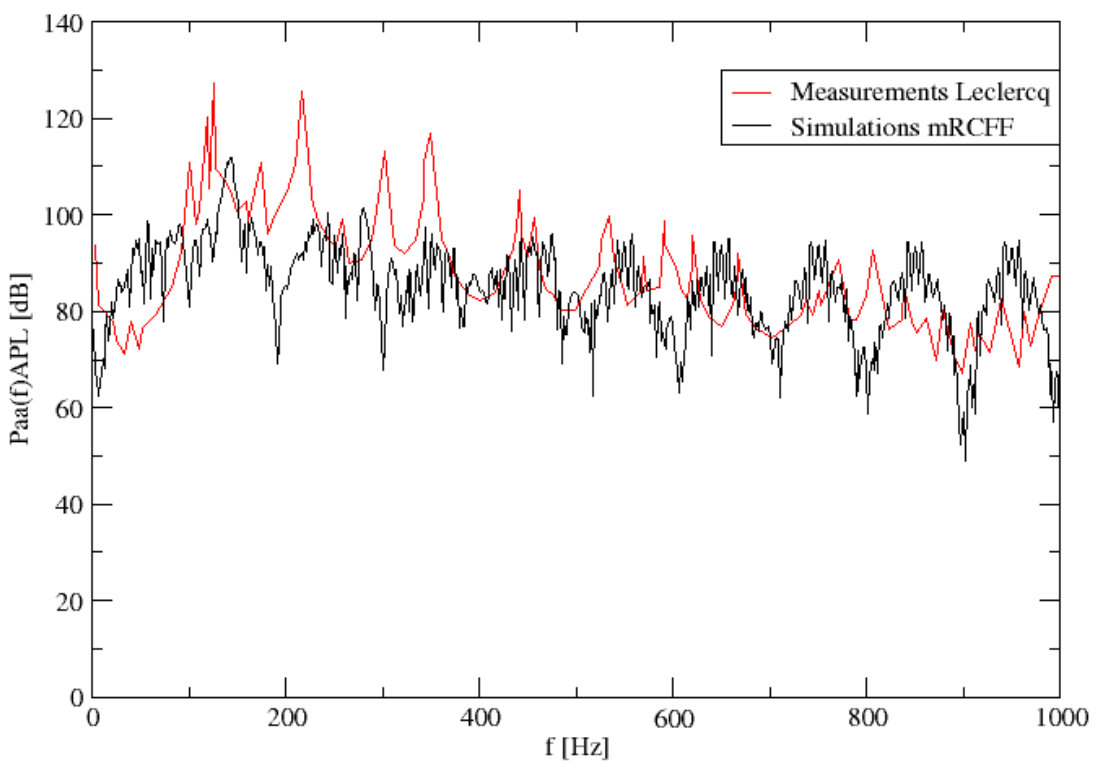

Figure 11 Power spectrum of acceleration, scaled with $1 \mathrm{e}-6 \mathrm{~m} / \mathrm{s} 2$

Figure 11 shows the acceleration of a point on the plate. The simulated levels and the spectrum shape compare rather well with the measurements by Robinson et al (1993). However, there are higher peaks in the measurements. As the pressure levels at lower frequencies compare rather well, see Figure 9 above, the underestimation of the acceleration could be due to deficiencies in solid modeling. The numerical damping in the solid is probably overestimated, which can have an impact on the acceleration. 


\subsection{Assessment Case 2}

The methods are assessed as sufficient to study how a structure is affected by the pressure due to turbulent fluctuations, phenomenologically and to the order of magnitude.

The excitation of the plate in Case 2, with turbulent fluctuations, agrees well with simulations and measurements by Frendi and Robinson (1993) and Robinson et al (1992). Discrepancies in the damping are a likely cause of the differences that still exist, particularly over-estimated numerical damping in the solid. Other probable causes of errors are the sub-filter modeling and the solution accuracy, especially the time-scheme, in the fluid.

Previous simulations and analyses, see Lillberg and Nilsson (2008), have shown that the convective boundary condition used at the outlet can reflect some acoustic energy. The energy may therefore be wrongly kept in the domain and thus provide elevated levels of background noise. Such a thing cannot be seen the results here. This suggests that sufficient acoustic energy is allowed to propagate, but it can also be due to that the corresponding energy is dissipated in the fluid domain.

The flow structures that appear to create the most significant pressure fluctuations are the rolls that are created after the ruler. They are quite large, of the order of $10 \mathrm{~mm}$. At the same time, the plate deflection is of the order of less than $0.1 \mathrm{~mm}$, see Figure 8 . Therefore it is considered unlikely that the plate deformation would affect the flow field, so that the generation of the dominant pressure fluctuations would be affected. Possibly a small effect can be seen at low frequencies in Figure 9, but in principle the FSI is weakly coupled.

\section{CASE 3}

In Case 3, the excitation of a structure in the region around nuclear reactor steam dryers is studied. The background is the events at the power uprates in the reactors at Quad Cities and Dresden, see for example Hambric et al (2006). These have attracted justified attention in Sweden, where several power uprating projects are running. For other related analyses, see for example Strauss (2005).

For this purpose a computer model, which resembles the steam parts of the reactor in Quad Cities I, has been created. This particular plant was chosen because a dryer cover plate was excited there. Since the exact specifications of reactor components are rarely public, the design and measures have been estimated from measurements and drawings in public material; see Hambric et al, Henry et al and Wellstein et al. The eigenfrequencies of the cover are hard to assess, because they depend strongly on how the plates are connected and the design of the internal structures. For a $6.35 \mathrm{~mm}$ steel plate of this size, the first eigenfrequency may however lie below $20 \mathrm{~Hz}$.

The simulations including the plate structure are not finished, so only results from flow simulations are shown here. Discretization sensitivity has also not yet been explicitly tested for this case.

\subsection{Boundary conditions}

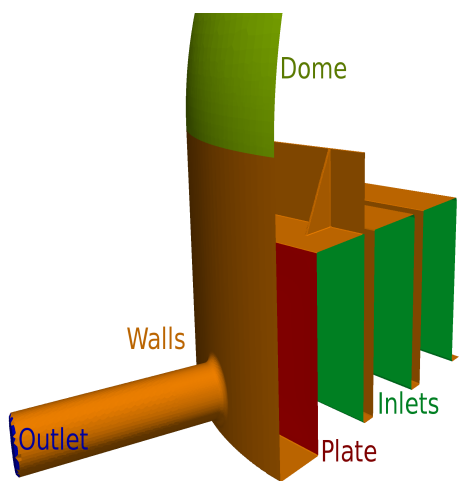


Figure 12: Calculation domain. The red plate just inside the steam outlet was excited in Quad Cities I.

Figure 12 provides a schematic of the domain. All walls in geometry, with the exception of the spherical dome, are treated as walls. Slip boundary conditions are used on the dome wall and no-slip on the other walls. The inlet is specified as a constant mass flow of $360 \mathrm{~kg} / \mathrm{s}$ (which gives an average speed of $0.78 \mathrm{~m} / \mathrm{s}$ at a temperature of $583 \mathrm{C}$ ), while the outlet is controlled by a constant total pressure of 6.9MPa.

\subsection{Numerical model}

Several different calculations have been made with the compressible LES-solver coodles. Different regions of the mesh have been refined, for example around the steam exit nozzle. The final calculation mesh, which was generated using OpenFOAMs snappyHexMesh, consists of $4.5 \mathrm{M}$, mainly orthogonal, hexahedra. This is considered to provide a sufficient resolution of large-scale free stream structures in the interesting region in front of the plate. The volume layer on the plate is $1 \mathrm{~mm}$ thick. This is not sufficient for a normal boundary layer at these flow speeds, but is considered reasonable here, because it will be shown that the flow is dominated by detachment at sharp edges of the geometry. Especially the top of the skirt creates large-scale vortex structures across the plate. For Case 3 , the time step was $2 \mathrm{e}-5 \mathrm{~s}$, resulting in a convective CFL ratio of 0.5 based on the volume size and flow velocity.

\subsection{Flow results}

The volume in front of the plate is the focus of this analysis. The flow there is dominated mainly by three factors: (i) the flow field from the upper part of the tank, (ii) the separation line along the top edge of the plate, and (iii) the outlet through nozzles.
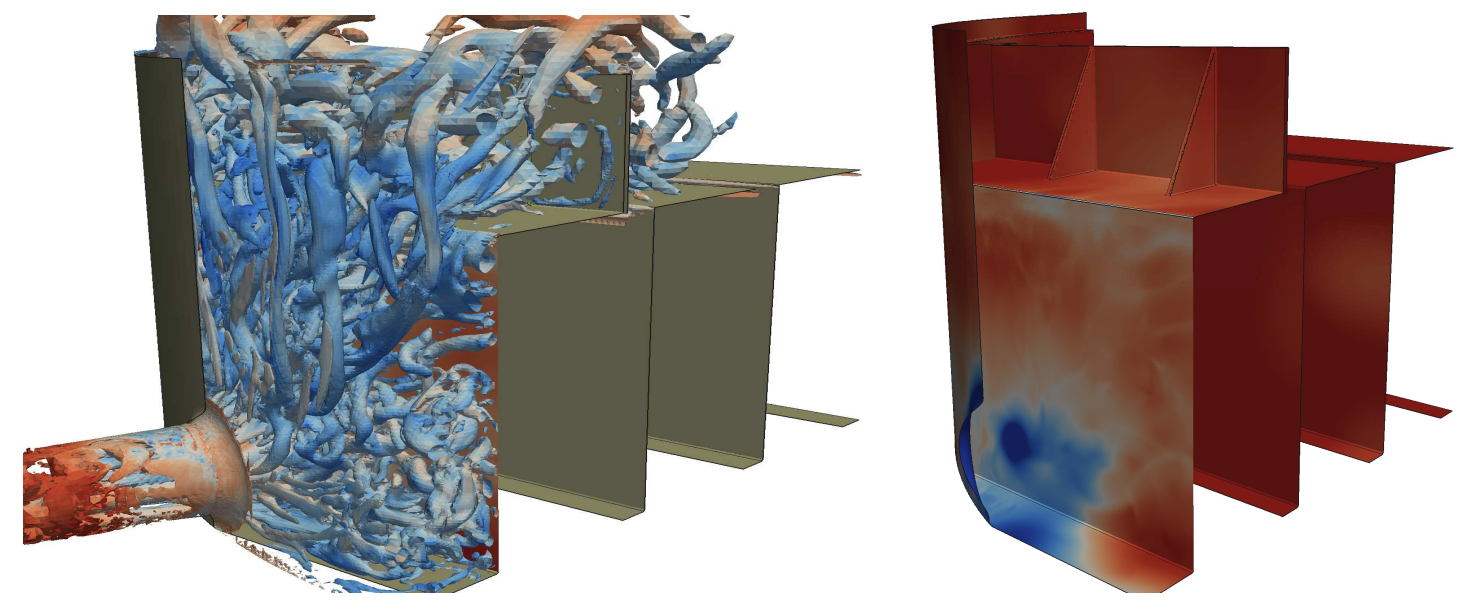

Figure 13: Isosurfaces of Q colored (left) Pressure on walls (right)

The turbulent flow is dominated by longitudinal vortex structures together with transverse vortices which separate at the plate edge, see Figure 13 (left). When the steam goes out through the outlet nozzles, the vortical structures are stretched and strengthened as they are oriented along the outlet.

There is a strong swirl generated at the outlet nozzle. This swirl creates a low pressure region at the plate, see the blue region in Figure 13 (right). The location of the vortex can be described as semistationary; it wanders around a center point.

\subsection{Spectrum of turbulent kinetic energy}

A spectrum of the turbulence energy gives information if the model is sufficiently resolved to give reliable results. The highest possible frequency of spatial oscillations is calculated as the mean velocity in a region, divided by the size of the volumes there. Over the plate, the velocity is about $20 \mathrm{~m} / \mathrm{s}$ and the volume size is about $10 \mathrm{~mm}$. Using Nyqvists theorem, this gives that fluctuations up to $1 \mathrm{kHz}$ are supported. Energy above this should be dissipated by the turbulence model. Figure 14 shows 
the energy content at two points, denoted 1 and 5 . Above about $1 \mathrm{kHz}$, the energy levels fall one to two orders of magnitude, which suggests that the LES model works well in that aspect.

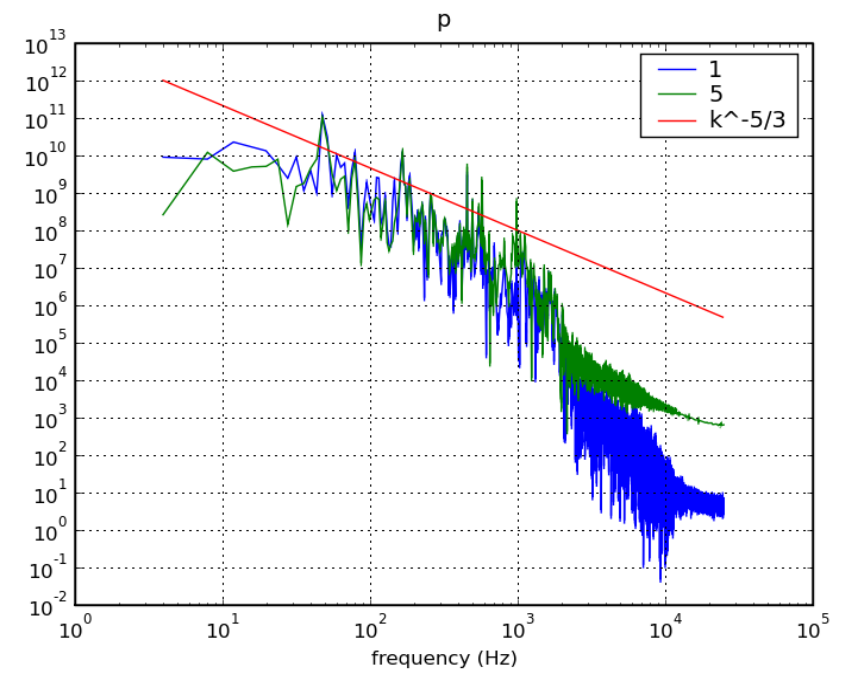

Figure 14: Power spectrum of pressure in some points before the plate

\subsection{Assessment case 3}

Based on previous discussions on large-scale separation and the relatively large energy-containing structures, it is concluded that the plate vibrations about one millimeter hardly can create large changes in the flow field. On the other hand, if the geometry had lacked sharp edges in the regions of detachment, small changes in geometry and vibration could play a crucial role for the flow field.

The pressure spectrum in Figure 14 shows some coinciding peaks for points 1 and 5, but only the peak at about $50 \mathrm{~Hz}$ is clear. Such a peak could match an eigenfrequency of the structure (as they are estimated to start at about $20 \mathrm{~Hz}$ ), but this is hard to assess at this stage with the available design data.

There is a slight possibility that the wandering around and fluctuations of the low pressure region inside the nozzle could excite the plate, but since the deflection is still small relative to the flow structures, it is unlikely that there would be a two-way FSI.

\section{COMMENTS ON THE SOFTWARE}

The solver for the solid is based on a formulation that is suitable for flow problems. It solves the equations for one direction at a time, $\mathrm{x}, \mathrm{y}$ and $\mathrm{z}$. For a problem like this it is not optimal, since the deformation of a solid is much stronger coupled in the three directions. To address this issue, very strict convergence criteria are set with a large number of iterations.

The boundary conditions used at the outlet are non-reflective to small, perfectly parallel disturbances. For other waves there is reflection. This keeps noise in the domain and likely causes error in Case 2.

Earlier tests have shown that the first-order scheme is a bit too diffusive for this type of aero-acoustic problems. Only Euler and backward-time schemes are available for moving grids in this version of OpenFOAM. The backward scheme is not limited but may lead to overshoots, dispersion.

It is presently not possible to use more than one fluid domain in a parallel solution. This is the reason for connecting the upper and lower fluid domains in Case 2.

In the current solver, the mesh is updated in all directions at all points of the domain. This is obviously not needed for such a local and one-directional deformation as we have here. The solver improvement is however not straightforward, without losing the generality of the regionalization and parallelization. 


\section{CONCLUSIONS}

It is possible to simulate structural excitation due to both sound and turbulence using the reported CFD methods, at least qualitatively. The excitation levels are also of the correct order of magnitude in some parts. There are some obvious shortcomings in the modeling. One is the lack of non-reflecting boundary conditions. Another important aspect is the strong numerical damping in combination with the demanding numerics of solid solution. More sensitivity studies are also needed to determine the quantitative accuracy of the predictions, particularly in Case 3 which is not compared to experiments.

One goal of this work was to study how well a fairly standard methodology, based on open source software and methods for FSI which is common in commercial CFD codes, performs at comparisons with experiments. It is an attempt to highlight the many difficulties with the methodology and some numerical aspects which may substantially affect the results in different ways.

Another objective of this work was to determine whether acoustic loads and flow loads can interact in such a way that the excitations can be stronger than with each load separately. For the cases tested here, especially Case 3, it is unlikely that the mechanisms of excitation can interact. The main reason for this is that the flow is controlled more by the static geometrical factors, such as solid rotation sharp edges, than small deformations due to vibrations.

As a side effect, another possible excitation mechanism was found; the low pressure region wandering around inside the steam exit nozzle. This phenomenon is however not coupled FSI.

\section{REFERENCES}

S.A. Hambric, T.M. Mulcahy, V.N. Shah, T. Scarbrough and C. Wu, "Flow Induced Vibration Effects on Nuclear Power Plant Components Due to Main Steam Line Valve Singing", Proceedings of the Ninth NRC/ASME Symposium on Valves, Pumps and Inservice Testing 2006. (NRC NUREG CP-0152 vol. 6)

D.O. Henry, L.K. Liu, J. Lynch, D.C. Pappone, M.R. Patel, S. and Sundaram, "Technical Assessment, Quad Cities Unit 2, Steam Dryer Failure - Determination of Root Cause and Extent of Condition", General Electric non-proprietary GENE-0000-0018-3359-NP, revision 0 (NRC ADAMS XR16591702)

L. Wellstein, D. Pappone, A. Pinsker, T. Le, M. Hayes, J. Waal, and B. Knott, "Quad Cities Units 1 and 2 Replacement Steam Dryer Analysis Stress, Dynamic and Fatigue Analyses for EPU Conditions", GE-NE-0000-0034-3781, Revision 0 (NRC ADAMS XR18384308)

J. Strauss, "CFD Modeling of the Vermont Yankee Steam Dryer Phase - II, Fluent Inc. Final Report" TM-675, 2005. (NRC ADAMS ML051590217)

A. Frendi, and J. Robinson, "Effect of Acoustic Coupling on Random and Harmonic Plate Vibrations", AIAA Journal, Vol. 31, pp1992, 1993.

J.H. Robinson, S.A. Rizzi, S.A. Clevenson and E.F. Daniels, "Large Deflection Random Response on Flat and Blade Stiffened Carbon-Carbon Panels", AIAA Paper 92-2390, 1992.

S. Vergne, J.-M. Auger, F. Périé, A. Jacques and D. Nicolopoulos, section 6.4 "Aeroelastic Noise", in Wagner, C., Hüttl, T. and Sagaut, P. (editors), "Large-Eddy Simulation for Acoustics",

Cambridge University Press, 2007.

D.J.J. Leclercq, "Modélisation de la réponse vibro-aqoustique d'une structure couplée à une cavité en présence d'un écoulement turbulent", PhD Thesis, Deptartement Génie Mécanique, Université de Technologie de Compiègne, France, 1999.

OpenFOAM, The Open Source CFD Toolbox, www.opencfd.co.uk.

E. Lillberg and P. Nilsson, "Generic CFD for Vortex Induced Acoustic Resonance in Deep Cavities", $16^{\text {th }}$ International Conference on Nuclear Engineering, ICONE 16-48173, 2008.

\section{ACKNOWLEDGEMENT}

This work was supported by the Swedish Nuclear Radiation Authority. 